\title{
Lire la peinture, lire le texte littéraire à l'école : une activité de même nature?
}

"Image reading" and analytical reading of literary texts: the same cognitive process?

\section{Marie-Sylvie Claude}

\section{OpenEdition}

\section{Journals}

Édition électronique

URL : http://journals.openedition.org/educationdidactique/2468

DOI : 10.4000/educationdidactique.2468

ISSN : 2111-4838

\section{Éditeur}

Presses universitaires de Rennes

Édition imprimée

Date de publication : 30 mai 2016

Pagination : 67-76

ISBN : 978-2-7535-5060-5

ISSN : 1956-3485

\section{Référence électronique}

Marie-Sylvie Claude, «Lire la peinture, lire le texte littéraire à l'école : une activité de même nature? »,

Éducation et didactique [En ligne], 10-1 | 2016, mis en ligne le 30 mai 2018, consulté le 20 avril 2019.

URL : http://journals.openedition.org/educationdidactique/2468 ; DOI : 10.4000/

educationdidactique.2468 


\title{
LIRE LA PEINTURE, LIRE LE TEXTE LITTÉRAIRE À L'ÉCOLE : UNE ACTIVITÉ DE MÊME NATURE?
}

\author{
Marie-Sylvie Claude \\ ESPE de Créteil, Laboratoire CIRCEFT-ESCOL
}

\begin{abstract}
Les programmes de français de collège et de lycée invitent à pratiquer la lecture de l'image, présupposant l'isomorphisme avec la lecture analytique des textes, dont elle favoriserait l'apprentissage. Notre recherche confirme que l'exercice appliqué à la peinture favorise l'accès aux attendus scolaires pour la majorité des élèves et suscite moins d'inégalités de réussite. Ce que nous expliquons par les écarts sémiologiques entre les deux arts et par le rapport différencié des élèves à l'un et à l'autre. Postuler l'activité de construction du sens comme de même nature risque donc de courtcircuiter les causes de la facilitation et, partant, la possibilité d'un transfert des apprentissages d'un art à l'autre.
\end{abstract}

Mots-clés : art, littérature, transfert de connaissances, égalité des chances.

"Image reading" and analytical reading of literary texts: the same cognitive process?

French secondary school curricula encourage the practice of "image reading", assuming isomorphism with analytical reading of literary texts, thereby stimulating its learning process. Our research confirms that the practice applied to paintings' analysis helps most pupils to meet academic standards and leads to fewer inequalities in academic success. We explain this by the semiological differences between the two Arts and the differentiated relation of the pupils to each of them. Postulating the sense-building activity as of the same kind is therefore likely to bypass the causes of the facilitation process and consequently the chance of transferring the learning process from one Art to the other.

Keywords: art, literature, knowledge transfert, equal opportunity. 
Cet article s'appuie sur une recherche consacrée aux liens entre la lecture littéraire et un exercice souvent préconisé de lecture de l'image, dénomination qui nous semble poser problème si elle est prise au pied de la lettre, car elle indifférencie alors deux langages hétérogènes : si les éléments plastiques, qu'ils forment ou non des ensembles figuratifs, peuvent être considérés comme des signes puisqu'ils sont porteurs de sens, ils ne relèvent pas d'un système d'encodage linguistique. Louis Marin (1971, p. 20), revenant sur l'injonction de Poussin à Chantelou dans une lettre du 28 avril $1639^{1}$ « lisez l'histoire et le tableau, afin de connaître si chaque chose est appropriée au sujet », analyse l'expression comme une invitation à une "lecture plastique» du tableau mais définit cette lecture dans ses écarts avec celle du texte : la peinture génère bien du lisible, mais il ne s'appréhende pas comme le lisible verbal. Pour rester ici dans le domaine de la peinture, le peintre fait des choix de combinaisons et de dosages possibles dans le vivier de ses moyens propres - composition, graphisme, teintes, valeurs, textures... : ce sont les effets de sens qui en résultent que le spectateur qui souhaite commenter la peinture a pour rôle de co-construire. Cette pratique peut certes être scolarisée, à l'instar du commentaire littéraire ${ }^{2}$. Mais les deux objets faisant sens différemment, les élèves peuvent-ils réaliser, en travaillant sur l'un, des apprentissages susceptibles de profiter à l'autre ? La préconisation de faire de l'image une médiation vers la lecture littéraire, ce qui présuppose la possibilité d'un transfert de compétences d'une activité à l'autre, se trouve, en France, par exemple, dans les programmes de français du collège, qui conseillent la lecture de l'image pour « consolider l'apprentissage de méthodes d'analyse » du texte ${ }^{3}$; elle est promue dans un rapport de l'inspection générale de 2000, selon lequel « la pratique de l'image constitue un apport méthodique indéniable, dans la mesure où la démarche d'analyse pour "déchiffrer" une image est plus spontanément reçue par les élèves » (Waysbord-Loing, p. 16), notamment par ceux qui sont peu pré-familiarisés avec la culture littéraire. Dans le cadre de la recherche en didactique, la question est notamment envisagée par Annie Rouxel (1996), qui écrit que « la lecture d'une photographie, d'un tableau ou d'une sculpture repose sur les mêmes opérations mentales que la lecture d'un texte », mais « sollicite les affects différemment » de sorte que « du transfert des compétences qui s'opère en passant d'un art à l'autre, on escompte leur mise à l'épreuve et leur renforcement. » (p. 128-129.) Nous avons souhaité interroger cette hypothèse en analysant de façon comparative des commentaires écrits sur l'un et l'autre des deux objets par des élèves de troisième et de seconde. Nous préciserons le cadre de notre recherche puis présenterons, à titre d'exemple, l'analyse des deux commentaires du même élève, avant de proposer quelques pistes d'explication des différences observées et de tenter d'en préciser les incidences pédagogiques.

\section{CADRE THÉORIQUE}

Nous inscrivons notre travail dans le cadre plus général de l'étude des effets des inégalités sociales sur les apprentissages, interrogés eu égard aux activités spécifiques qu'ils requièrent. Nous avons tenté de croiser sociologie de l'éducation et didactique afin de savoir si, compte tenu des spécificités des deux activités, le commentaire de la peinture pouvait favoriser l'apprentissage du commentaire de la littérature, et si c'était le cas semblablement pour tous les élèves.

Le modèle des registres de l'apprentissage (cognitif, culturel et symbolique), travaillé par l'équipe ESCOL, nous a permis de déconstruire l'activité sous-tendant les commentaires, afin d'en comparer les composantes, pour chaque élève, selon qu'il commente la peinture ou la littérature : les opérations cognitives, les savoirs mobilisés et les caractéristiques "identitaires symboliques », c'est-à-dire celles qui concernent l'engagement du sujet et les liens symboliques que par son travail il établit avec autrui ${ }^{4}$, sont plus ou moins conformes aux attendus des enseignants. Les travaux de la didactique de la lecture littéraire nous ont aidé à comprendre les composantes de ces trois registres en commentaire littéraire, les apports de l'histoire de l'art et de la sémiologie picturale nous ont été nécessaires pour tenter de transposer cette compréhension aux particularités du commentaire pictural. Ce double apport, croisé avec la notion de « malentendu sociocognitif $»^{5}$, issue de la sociologie des apprentissages, nous a en outre permis de proposer des pistes d'explication de ce que nous observions. 


\section{MÉTHOdOLOGIE}

Nous avons fait rédiger à 350 lycéens et collégiens de l'académie de Créteil deux brefs commentaires, l'un sur une reproduction picturale et l'autre sur un extrait littéraire ${ }^{6}$. Les conditions de travail étaient les mêmes pour les deux supports et la consigne était rédigée semblablement : "écrivez dix lignes (au minimum) pour commenter (donnez quelques-unes de vos impressions) $\gg$. Suivait une phrase autorisant à ne pas traiter l'un des deux objets en expliquant pourquoi. Nous avons choisi des établissements aussi différenciés que possible quant aux catégories socio-professionnelles composant le public qu'ils accueillaient. Nous avons complété ce matériau par des entretiens post-passation avec 11 groupes de 2 à 4 élèves issus des établissements les plus défavorisés.

Pour disposer d'une référence à l'aune de laquelle comparer les commentaires, nous avons obtenu de 108 enseignants de français de l'académie de Créteil qu'ils répondent à un questionnaire portant sur leurs attendus. Ceci nous a permis, en dégageant des consensus, d'avoir accès à ce que nous appellerons les registres attendus, qui relèvent majoritairement, sur l'un comme sur l'autre des deux objets, de la transposition d'une approche sémiotique (au sens d'Umberto Eco, 1990). Cette enquête fait apparaître une forte adhésion des enseignants concernés à l'hypothèse d'une facilitation, par la peinture, de l'accès aux compétences requises par le commentaire littéraire.

\section{RÉSULTATS}

Les élèves sont sensiblement plus nombreux à ne pas commenter le texte $(30,5 \%)$ que la peinture (5,5\%). L'écart est plus net encore si nous ne retenons que les travaux des élèves scolarisés dans des collèges de recrutement défavorisé ( $1,5 \%$ ne traitent pas la peinture et $47,5 \%$ ne traitent pas la littérature). Ils ne sont donc pas déconcertés par la transposition à la peinture d'une consigne à laquelle ils sont plus habitués, en tout cas en classe de français, sur le texte, et ont vraisemblablement le sentiment d'une meilleure compétence, convoquant sans doute des savoirs et savoir-faire acquis en histoire des arts ou en arts plastiques tout en s'en tenant à la forme attendue en français.

Ceci ne veut cependant pas dire que les registres mobilisés par les élèves y sont toujours plus compatibles avec les registres attendus. Pour évaluer de façon comparative d'un objet à l'autre, pour chaque élève, ce degré de compatibilité, nous avons déterminé, d'après les réponses des enseignants, une série de critères définissant chaque registre attendu. Puis nous avons recherché les caractéristiques langagières qui nous permettaient, pour chaque critère, de classer chacun des commentaires dans un type plus ou moins proche des attendus.

\section{UN EXEMPLE}

Pour donner une idée aussi précise que possible de notre travail, dans un format succinct, nous nous
Cette peinture créée par George de La Tour entre 1645 et 1650 est aujourd'hui exposée au Musée des Beaux Arts de Rennes.

On peut voir sur cette peinture trois personnages, deux femmes et un enfant. George de la Tour a peint la femme qui se trouve au premier plan d'habit plutôt sombre avec quand même un petit chemisié blanc pour qu'elle puisse attiré le regard. Au second plan nous pouvons voir une femme habillée de rouge tenant dans ses mains un enfant envelopé dans un drap blanc. Le nom de l'œuvre nous permet d'affirmé que cet enfant est un nouveau née.

Dans cette Euvre George a beaucoup joué avec la lumière en opposant la clareté des habits blancs avec le décort obscure du fond de la pièce. George de la Tour nous montre ici beaucoup de couleurs qui s'opposent tel que le blanc et le noire. L'auteur a joué aussi sur l'opposition du rouge et du blanc, le rouge représentant la mort, les enfers et le blanc lui représente la nativité, le paradis. Sur cette œuvre aucune ligne, aucune information pouvant déterminer un point de fuite de l'auteur. Dans l'œuvre l'auteur a utilisé la lumière pour attirer le regard du spectateur vers la clareté vers le bébé.

Pour moi cette œuvre est une belle représentation de la nativité avec comme commentaire de l'auteur que derrière ce bonheur qu'est la natalité, se cache une certaine froideur ce fond sombre représentant l'inconnue.
Cette pièce est une pièce de théâtre écrite par Molière en 1673. Cette pièce est une comédie.

Dans cette pièce Toinette se fait passé pour un médecin très expérimenté dans le but de discridité le médecin d'Argan.

Toinette vas se faire passé pour un médecin de 90 ans à cette époque l'espérence de vie des hommes ne dépassait pas les 40 ans encore aujourd'hui la moyenne d'age n'est en dessous de 90 ans. Toitelle va multiplier les annerie envers son maître en lui faisant des diagnostiques n'ayant aucun rapport avec le symtome d'Argan. Cette pièce inspire le rire chez le spectateur / lecteur du fait de ses discréditation que Molière fais beaucoup dans cette œuvre ("Le médecin malgré lui »).

Molière discrédite aussi les bourgeois dans cette œuvre de par le fait qu'il gobent tout ce qu'on leur dit du moment que sa sort de la bouche d'une personne apparament forte dans son métier. 
Marie-Sylvie Claude

appuierons sur les commentaires de Jonathan, élève de troisième (sur le tableau de Georges de la Tour, $\mathrm{La}$ Nativité ou le Nouveau-né et sur un extrait du Malade imaginaire de Molière, acte III, scène 10).

\section{Le registre cognitif}

L'analyse des réponses que nous avons obtenues des enseignants montre qu'ils s'inscrivent toujours dans la «tradition scolaire » que décrivait Yves Reuter en 1992 : ils attendent qu'au-delà de ce qu'ils nomment la compréhension, qu'ils définissent comme la saisie d'un sens qui serait inhérent au texte, les élèves conçoivent un sens interprété, désigné comme un "surplus » ou un "surcroît» de sens. Ils attendent que ce sens soit pluriel et qu'il soit justifié par des données fines, de nature diverse, issues notamment de l'analyse de la forme de l'expression.

Sur le tableau, le commentaire de Jonathan émarge à certaines des caractéristiques langagières que nous avons définies pour repérer une telle démarche interprétative : notamment, le décalage entre le lexique de la description du tableau et un lexique s'en abstrayant (« mort », « enfers », " paradis », « bonheur », « froideur », « inconnue ») signale que le commentateur prend la responsabilité de la conception d'un "surcroît de sens ». S'articulent deux directions d'interprétation, la mise en valeur du nouveau-né et la présence d'une menace latente, justifiées par des composantes plastiques et iconiques, saisies selon des liens de complémentarité et d'opposition : d'abord, Jonathan constate que le contraste entre le clair et le sombre permet « d'attirer le regard du spectateur » sur la mère et le nouveau-né, désigné comme source de la lumière. Il travaille sur la composition singulière du tableau (« sur cette œuvre aucune ligne »), qui annule toute profondeur en même temps qu'elle efface tout décor et toute anecdote, le retrait de l'instance picturale (" aucune information pouvant déterminé un point de fuite de l'auteur $»$ ) concentrant toute l'attention du spectateur sur la contemplation du mystère de la nativité et de l'adoration qui habite les personnages. Une autre opposition chromatique, qui complexifie le clair-obscur, est ensuite analysée : celle du « rouge représentant la mort, les enfers », en contraste avec le blanc qui « lui représente la nativité ». La manifestation lumineuse du divin apparaît à Jonathan comme sous-tendue par une menace latente. Ce va-et-vient entre la désignation de composantes fines de l'objet et l'énoncé d'effets de sens est une autre des marques que nous avons retenues comme caractéristiques d'un registre cognitif compatible avec le registre attendu par les enseignants.

Sur le texte de Molière, l'activité cognitive nous semble différente : la démarche est interprétative au sens où le commentateur s'abstrait de l'univers diégétique pour concevoir la portée critique généralisante (de la ridiculisation d'Argan à la dénonciation d'un type social). Cependant, si Jonathan, « spectateur / lecteur », est sensible aux effets comiques des procédés langagiers et scéniques, dont il comprend en outre qu'ils participent de la satire sociale, il n'en déconstruit pas les composantes fines. Il s'en tient en effet à une appréhension générique : les éléments qui lui font apparaître comme telles " les annerie » de Toinette et suscitent « le rire » ne sont pas repérés. Les « discréditations » sont référées aux intentions et aux habitudes de Molière, mais non précisément aux effets de l'écriture théâtrale.

\section{Le registre culturel}

Le registre attendu est constitué des savoirs nécessaires pour comprendre ${ }^{7}$ le texte ou le tableau, mais aussi pour approfondir l'interprétation en croisant les données textuelles avec des « données externes » (Reuter $\left.{ }^{8}, 1992\right)$ et en travaillant sur les horizons d'attente ouverts par l'objet (Jauss, 1972) ou sur le sens des liens intertextuels (Genette, 1982) ou interpicturaux.

Sur le tableau, Jonathan ne reconnaît pas les personnages évangéliques. Ignorant la différence entre les mots nativité, natalité et naissance, il les emploie de façon indifférenciée et ne peut se saisir du titre pour étayer sa compréhension. Malgré son ignorance du thème iconographique, il en effleure cependant, par la seule observation du tableau, le contenu de sens spirituel : au bonheur d'Anne et Marie se mêle la prescience de la Passion. Jonathan devrait donc pouvoir aisément approfondir, à la lumière de la référence culturelle légitime, ce qu'il perçoit de la seule observation. Son intuition d'une représentation singulière de l'enfant, dont la divinité est tout entière contenue dans la lumière et dans l'adoration des Saintes Femmes, serait en outre consolidée par un savoir interpictural qui lui permettrait de donner sens à l'absence des attributs ordinaires de la Nativité 
dans ce tableau, qui met au cœur de la représentation le mystère de l'incarnation, le double titre étant révélateur du choix de représenter l'épisode évangélique comme une naissance ordinaire.

Sur le texte de Molière, Jonathan connaît le castigat ridendo mores, présent dans trois occurrences de la famille du verbe discréditer et vraisemblablement appris à propos du Bourgeois gentilhomme. Il convoque explicitement un intertexte, Le Médecin malgré lui. Cependant, le registre culturel n'intervient pas dans l'activité interprétative comme attendu par les enseignants : Jonathan ne décontextualise pas sa connaissance d'autres types comiques de manière à l'adapter à celui du malade imaginaire en s'appuyant sur les procédés langagiers et scéniques qui le constituent (effet d'accumulation des symptômes imaginaires, déférence excessive). Les connaissances scolaires, toutes légitimes qu'elles soient, sont plutôt exposées que mobilisées pour la justification de l'interprétation, et nous semblent de ce fait susceptibles de faire diversion et d'éloigner le commentaire des attendus.

\section{Le registre identitaire symbolique}

Les enseignants attendent que l'élève se constitue en sujet-lecteur, co-constructeur du sens; ils insistent sur l'engagement du sujet empirique, de ses émotions, qu'ils considèrent comme nécessaire pour nourrir l'interprétation, mais souhaitent que ce matériau soit ressaisi et retravaillé eu égard aux droits du texte (notamment : Rouxel \& Langlade, 2004). C'est le versant identitaire du registre, qui rejoint son versant symbolique, au sens où le sujet se relie ainsi à la communauté de réception de l'œuvre : l'attendu d'une reconfiguration de l'approche immédiate se justifie en effet par la nécessité qu'elle soit communicable à autrui. Pour l'objet qui nous occupe, jouer le jeu du commentaire suppose de surcroît l'acceptation de certaines valeurs lettrées : c'est en effet, d'après les réponses des enseignants, une conception très spécifique de l'œuvre d'art (signifiance des procédés formels, polysémie, ouverture à la réception...) qui induit la réception attendue à l'école, qui peut être très différente d'autres appréhensions possibles d'un texte, y compris littéraire, ou d'un objet visuel, y compris artistique, dans d'autres cadres. Il s'agit donc de s'inscrire dans les échanges verticaux constitutifs des apprentissages savants (Bernstein, 2007).
Le commentaire de Jonathan sur le tableau présente certaines des marques que nous avons retenues comme caractéristiques d'une conception de l'objet compatible avec les normes lettrées. Les mots « jouer sur » et « utiliser » manifestent la conscience claire que les procédés plastiques (et pas seulement iconiques) sont pour le peintre des outils d'expression et qu'ils peuvent faire signe pour le spectateur ; la formule " une belle représentation de la nativité avec comme commentaire de l'auteur » montre que la représentation picturale est comprise comme n'étant pas un enregistrement du réel mais le lieu d'une vision singulière, que le rôle du récepteur n'est pas de reconstruire mais de co-construire (la phase commence par «pour moi »). D'ailleurs, « l'auteur» n'a pas disposé de «point de fuite », de « lignes » de guidage : même si le peintre a des intentions, exprimées par les subordonnées de but, le sujet de l'énonciation, visible au travers des pronoms de la première personne et des modalisateurs, se positionne bien comme une instance sémiotique engagée dans le dialogue avec l'instance picturale (« nous pouvons voir », « nous permet d'affirmé »...).

C'est très différent pour le commentaire sur le texte de Molière, qui ne nous semble pas appréhendé comme le lieu d'un travail sur une forme virtuellement signifiante puisqu'aucune caractéristique stylistique n'est relevée ni aucune citation donnée à l'appui des effets de sens proposés. En outre, le seul élément de détail auquel il est fait référence concerne l'âge prétendu de Toinette, au tout début de l'extrait, comme si la lecture fine n'avait pas été plus loin. Jonathan semble penser qu'il doit aborder la pièce dans sa globalité (le mot "pièce » est employé cinq fois et le mot « œuvre » une fois), et s'appuie pour ce faire sur des informations présentes dans le paratexte, n'appréhendant vraisemblablement pas le début du texte de Molière comme un seuil langagier, ce qui est significatif d'un écart avec les conceptions majoritaires chez les enseignants, pour qui l'opposition avec le non littéraire est constitutive du texte littéraire. Par ailleurs, les marques de la première personne, qui saturaient le commentaire sur le tableau, sont ici absentes, le sujet de l'énonciation étant seulement désigné, de façon générique, par la périphrase scolairement correcte "spectateur / lecteur ». Le commentateur n'apparaît pas comme interagissant avec le texte pour en co-construire le sens.

Les deux commentaires de Jonathan sont donc sous-tendus par une activité très différente : le tableau 
est appréhendé de façon beaucoup plus minutieuse d'une part, et selon une attention à ses caractéristiques formelles (couleur et graphisme) d'autre part. La conception de l'objet et l'engagement du sujet y sont plus en phase avec les attendus. Cependant, les savoirs culturels légitimes font défaut sur le tableau plus que sur le texte. Ceci est représentatif des résultats que nous avons obtenus sur l'ensemble du corpus $^{9}$. En effet, pour le registre cognitif, 81,5\% des élèves produisent une interprétation sur la peinture (contre 60,5\% sur la littérature), 52,5\% donnent sens aux caractéristiques formelles (contre 18,5\%), $27,5 \%$ appréhendent l'œuvre comme un système signifiant complexe (contre $8 \%$ ), $59 \%$ comme polysémique (contre $22 \%$ ). Pour le registre identitaire symbolique, $54,5 \%$ des commentaires sur la peinture ne présentent pas d'écart perceptible avec les conceptions lettrées (contre $33 \%$ sur la littérature) ; $39 \%$ présentent un engagement subjectif ressaisi (contre $20 \%$ ). En revanche, pour le registre culturel, $57 \%$ seulement des élèves ne rencontrent pas de gêne - liée à des défaillances culturelles - pour la compréhension de la peinture (alors que c'est le cas de $73 \%$ des élèves pour la littérature), et $38 \%$ rencontrent une gêne marquée pour l'interprétation (contre $16 \%$ ). Les écarts se creusent nettement, pour chacun de ces critères, dans les collèges de recrutement défavorisé. Comment ces différences peuvent-elles s'expliquer?

\section{EXPLICATIONS}

Nous verrons d'une part que l'activité nécessaire pour satisfaire aux attendus n'est pas la même sur les deux arts parce qu'ils sont intrinsèquement différents ; d'autre part que le rapport des élèves aux deux arts n'est pas le même.

\section{Les différences entre les deux arts}

Un rapide rappel de quelques-unes des propositions théoriques de la sémiologie picturale déconstruit fermement l'évidence apparente sousjacente à l'expression lecture de l'image (Marin, 1971). Les écarts entre les deux langages ont des effets sur l'activité nécessaire au récepteur, donc à l'élève qui commente.

L'inexistence d'une langue picturale, qui codifierait de façon conventionnelle les corrélations entre forme de l'expression et sens, est un premier écart, essentiel. Le langage pictural est un langage dense (Goodman, 1968), constitué d'ensembles de couleurs et de traits, qui font sens sans conventions productives préétablies, du moins de type linguistique; chaque tableau de peinture élabore ses propres corrélations entre composantes plastiques et contenu en même temps qu'il élabore ce contenu (Eco, 1976). Contrairement au lecteur, le spectateur ne dispose donc pas d'un code linguistique associant des segments de l'expression à des segments de contenu; ce qui implique qu'il prend en charge, sans garantie, le sens qu'il donne aux signes. À la condition que l'élève entre dans le jeu de la construction du sens, ce qui n'est pas toujours le cas, le langage pictural est donc tel qu'il favorise la conception d'une interprétation, du moins au sens que les enseignants qui nous ont répondu donnent à ce terme, c'est-à-dire un « surcroît de sens» (qui relève du commentateur). Au contraire, l'élève peut avoir l'impression que le texte lui impose de s'en tenir à sa lettre ou lui permet de ne pas s'engager. Ainsi de Jonathan : quand il écrit que Toinette se fait passer pour un médecin pour se moquer de son maître, sa proposition est validée par le code linguistique; alors que quand il interprète la présence d'une menace inconnue derrière le bonheur lumineux émanant de la scène, il prend la responsabilité de cet effet de sens. Par ailleurs, les signes picturaux sont à la fois désignants (d'un figuré) et signifiants à valeur expressive (Marin, 1971), ils ont une double nature, iconique et plastique (Groupe $\mu$, 1992), ce qui peut expliquer pourquoi les élèves construisent plus souvent le sens en articulant les deux aspects du signe. Selon les conceptions lettrées, les signes verbaux sont eux aussi à appréhender comme faisant sens de leurs caractéristiques sensibles, mais l'existence du code linguistique peut masquer à une partie des élèves cette valeur. $\mathrm{Au}$ contraire, la peinture (c'est plus évident encore pour la peinture non figurative) incite son commentateur à inférer du sens des jeux de combinaisons, de parallèles et d'oppositions entre ses différentes composantes, donc à l'appréhender comme système signifiant complexe (donc selon les attendus des enseignants) ; ainsi de Jonathan, qui pourrait bien sûr épaissir le réseau des signes iconiques - main qui protège l'enfant et reçoit sa lumière, expression réservée des femmes etc. - et plastiques (géométrisation de la figuration, triangles des corps et ovales des visages, au service de l'expression de sérénité et 
de la grâce, etc.), mais qui initie bien la démarche attendue.

Une autre différence entre lire et voir, essentielle, réside dans l'initiative du spectateur quant à la temporalité de son appréhension : il lui revient de s'en tenir à un regard englobant ou d'organiser une successivité des éléments picturaux ; le tableau jalonne de façon plus ou moins contraignante ce parcours, mais il laisse toujours une part d'initiative au spectateur, même pour un tableau de facture classique (Marin). Ainsi du regard de Jonathan : il circule dans le tableau et revient sur les mêmes éléments, permettant un approfondissement progressif de l'interprétation ; il remarque d'ailleurs l'absence de lignes de construction ; on pourrait certes lui montrer que deux obliques lumineuses se croisent au sommet de la tête de l'enfant, y conduisant le regard ; pour autant, ce guidage par l'instance picturale peut être contourné. Certes, l'analyse d'un texte suppose aussi que l'appréhension n'en soit pas seulement successive ; cependant, la successivité s'impose à la première lecture. Le tableau de peinture rend donc à proprement parler visible la pluralité des approches possibles. Le processus même de construction du sens de la peinture, eu égard à ses caractéristiques propres et au type d'activité de commentaire qu'elles induisent, favorise donc, à condition que l'élève s'y engage effectivement, ce que sont justement, d'après notre enquête, les registres attendus des enseignants.

Par ailleurs, les différences intrinsèques entre les deux arts impliquent une grande exigence de nombreux tableaux de peinture sur le plan culturel et peut expliquer que, dans notre corpus, ce registre pose souvent difficulté aux élèves. L'élève lecteur de la littérature a besoin, même s'il ne suffit pas, d'un savoir linguistique, dont l'élève spectateur de la peinture peut se passer : pour autant, les signes picturaux produisent des corrélations culturelles, ils ne font pas sens naturellement, même s'ils ne sont pas en corrélation arbitraire avec leur contenu (Eco, 1976). Le meilleur exemple en est la connaissance, souvent nécessaire pour la peinture d'histoire, de l'iconographie. Erwin Panofsky $(1967)^{10}$ définit une progression par degrés de l'appréhension d'un tableau figuratif : le premier niveau, pré-iconographique, est celui de la reconnaissance de ce qui est figuré, c'est-à-dire des «motifs artistiques »; il permet d'appréhender les significations "primaires ou naturelles », dont Panofsky précise que notre "expérience pratique » (p. 17-18) nous suffit à les identifier et à en identifier la valeur expressive. Le second stade de la progression est celui de l'iconographie, par lequel le spectateur accède aux significations conventionnelles des motifs, dans lesquels il reconnaît les thèmes ou concepts (cet homme et cette femme nus sont Adam et Eve, ce paon est l'attribut d'Héra...). Des références culturelles, parfois savantes voire très savantes, y sont nécessaires, ce qui peut mettre en difficulté les élèves : ainsi Jonathan est-il tout à fait capable de travailler finement sur le plan pré-iconographique ; mais, du fait qu'il ne reconnaît pas le thème de la Nativité (comme $74 \%$ des élèves de notre corpus), son interprétation ne peut être considérée comme satisfaisant aux attendus car elle n'est pas recevable en l'état dans une communauté lettrée, qui connaît le sens symbolique dont la culture commune a doté certains motifs. La connaissance des contenus de sens liés aux thèmes iconographiques serait à même de nourrir le commentaire : Jonathan pourrait y trouver de quoi expliquer la menace qu'il perçoit dans le fond noir (prescience de la passion) et donner sens à la sobriété de la représentation (Jésus comme fils de l'homme). Hormis les conventions iconographiques, certains figurés ou certaines configurations plastiques sont associés, préalablement à leur réalisation dans le tableau, à des contenus de sens, qui, s'ils n'épuisent jamais l'interprétation - le contenu d'un tableau ne peut pas s'épeler (Arasse, 2009) - sont souvent utiles à la conception d'une interprétation recevable par une communauté lettrée, dont on suppose qu'elle dispose de ces savoirs : valeur symbolique conventionnellement attachée, dans un contexte donné, à telle ou telle composante, plastique ou iconique (couleurs, objets, geste, posture, mimique...) ; ou contenu de sens que peut prendre, dans l'œuvre d'un peintre particulier, tel motif ou telle configuration plastique récurrents.

La peinture est donc telle, par ses caractéristiques propres, qu'elle exige souvent des savoirs culturels savants mais qu'elle est susceptible de faciliter la réalisation des opérations cognitives telles qu'elles sont attendues des enseignants; mais c'est à la condition que l'élève s'engage effectivement dans la construction du sens; or cet engagement est coûteux : il incombe au commentateur de prendre l'initiative de la durée et du parcours de son regard et la responsabilité des relations qu'il fait entre caractéristiques du langage plastique et effets de sens, alors même qu'elles peuvent paraître peu motivées. Il nous reste donc à expliquer l'acceptation de cet engagement. 


\section{Des attendus mieux entendus}

Nous avons précisé, à propos des résultats que nous avons obtenus concernant le registre identitaire symbolique, que, d'après leurs commentaires, les élèves accèdent plus souvent sur la peinture que sur la littérature aux normes lettrées : c'est-à-dire qu'ils acceptent le visible artistique comme devant faire l'objet d'une réception très spécifique plus souvent qu'ils ne le font pour le lisible littéraire. Or cette acceptation est d'autant plus exigeante en termes d'adhésion symbolique que, dans les deux cas, rien ne permet, dans l'absolu, de reconnaître l'objet comme artistique ou littéraire, ni comme devant faire l'objet d'une appréhension spécifique du fait de cette articité : cette catégorisation est le fait non de la nature de l'objet mais d'une construction culturelle lettrée, dont relève aussi la définition de la réception à laquelle il doit donner lieu. Il est par conséquent indispensable que l'élève soit en mesure d'entrer dans les normes de cette communauté lettrée, sans quoi l'exercice ne peut que lui paraître opaque voire absurde ; en fonction de leur socialisation antérieure, certains élèves y accèdent par imprégnation culturelle, alors que pour d'autres, un apprentissage spécifique serait nécessaire. Or un tel objectif n'apparaît ni dans les programmes ni dans les réponses que nous avons obtenues des enseignants : pour la littérature, les statuts textuels et les types de lecture pratiqués y apparaissent souvent comme "naturalisés » (Reuter, 2001) ; pour la peinture, rien n'est dit hormis le parallèle avec la littérature. Tout se passe comme si le partage des implicites devait aller de soi : quand les élèves entendent mal les conceptions lettrées, cette difficulté est donc co-construite, on peut parler de malentendu. Or notre analyse des commentaires fait apparaître, pour ce qui est de la littérature, des écarts avec les conceptions lettrées pour $81,5 \%$ des commentaires dans les collèges à recrutement défavorisé et pour seulement $57 \%$ des commentaires dans les autres collèges. Le malentendu avec les enseignants concernant la conception de la littérature serait donc sociocognitif, puisque les différenciations sociales ont un net effet. La peinture en est d'après nos résultats bien moins affectée que la littérature: l'écart entre satisfaction aux registres attendus sur la littérature et sur la peinture, en faveur de cette dernière, est bien plus creusé dans ces collèges.

Pour approfondir le caractère sociocognitif du malentendu sur la littérature, il nous faut nous demander pourquoi il est moindre sur la peinture. Il nous semble que ceci peut s'expliquer, entre autres facteurs, par le fait que langage verbal est celui des interactions quotidiennes; or dans ce cadre, il a peu en commun avec le langage de l'école en général et encore moins avec le langage littéraire tel que l'école le définit. Le " rapport scriptural-scolaire au langage » (Lahire, 2008, p. 55), rapport réflexif, est très différent de celui qui est pratiqué dans la vie courante, à l'oral ou dans des formes d'écriture peu élaborées, où le langage est un outil inconscient, transparent, d'interactions sociales. À l'école, l'élève doit se mettre « hors-jeu par rapport à ses pratiques langagières ordinaires » (p. 29). Ce qui est vrai pour toute pratique langagière scolaire l'est sans doute plus encore pour la lecture littéraire : non seulement le langage doit y être perçu comme objet à observer et à réfléchir mais encore, il doit être appréhendé comme ne transmettant pas directement une signification qui suffirait à sa lecture puisqu'il faut inférer du sens (sens de surcroît présumé comme pluriel) des agencements particuliers de la forme de l'expression. La polyfonctionnalité du langage verbal nous semble donc susceptible d'engendrer des malentendus pour les élèves les moins coutumiers de la variété des usages possibles du langage. La peinture, qui n'est pas de langage verbal, en est partiellement épargnée : quand bien même le langage iconique est très présent, ce n'est pas le langage privilégié des interactions quotidiennes.

Surtout, ce n'est pas le métalangage du commentaire. Or, dans les entretiens post-passation, la récurrence est frappante d'une conception selon laquelle le texte étant déjà écrit, son récepteur n'aurait pas de place pour son propre écrit. Quelques exemples : « sur le tableau il y a rien d'écrit, c'est à nous d'écrire » (Amélie, troisième) ; « une peinture... il y a pas de texte... je veux dire c'est nous on imagine... alors qu'un poème il y a des textes... » (Dayane, seconde). « Dans les textes on a des mots à comprendre alors que sur un tableau c'est nous qui allons dire notre... allons écrire...» (Kourosh, seconde). Selon cette conception, la peinture laisse de la place à l'écriture du commentateur car son texte n'est pas encore écrit, il est comme en attente de l'être par nous - ce qui est une traduction acceptable de la conception lettrée sous-tendant les attendus enseignants - alors que pour la littérature, la place serait en quelque sorte déjà prise. Or, dans la conception lettrée, le commentateur doit co-construire le sens de la littérature tout 
Marie-Sylvie Claude

autant que de la peinture : son métatexte n'est pas en concurrence avec le texte commenté même si le langage verbal leur est commun. La peinture préserve donc de la confusion entre deux usages du langage, le langage du commentaire et celui du texte, que l'absence de familiarité avec les normes de la culture lettrée interdit à une partie des élèves de discriminer en tant que deux usages du langage verbal considérés dans la culture lettrée comme fondamentalement différents.

\section{CONCLUSION}

D'après notre expérimentation, les registres en œuvre dans les commentaires des élèves sont plus en phase avec les registres attendus des enseignants sur la peinture que sur la littérature, du moins pour le registre cognitif et le registre identitaire symbolique. De plus, l'écart entre les deux objets se creuse dans les établissements de recrutement populaire. Ce sont les caractéristiques sémiologiques de la peinture qui expliquent, d'après nous, que, par rapport à la littérature, elle favorise l'accès aux opérations cognitives attendues. La différence du matériau métalangagier avec le langage de l'objet réduirait en outre les occasions de malentendu pour les élèves les moins familiers des normes lettrées. Ce sont donc les écarts entre les deux objets qui, générant une différenciation de l'activité requise pour construire leur sens, peuvent faire de leur scolarisation en parallèle une ressource pédagogique. De sorte que présupposer l'isomorphisme entre les deux activités risquerait d'en faire échouer le profit escompté. Pour assurer le transfert des compétences de l'un à l'autre des deux objets, un cadrage explicite des échos et des écarts entre les deux activités attendues nous semble nécessaire, sans quoi il est à craindre que les malentendus restent entiers.

\section{NOTES}

1. Accompagnant l'envoi du tableau Les Israelites ramassant la manne du désert.

2. Nous utiliserons le mot commentaire sans le limiter à l'exercice du baccalauréat en tant que forme rhétorique, mais pour désigner le genre scolaire de l'écriture interprétative métatextuelle (Daunay, 2004) pratiquée sur un texte littéraire - ou posé comme tel - et son adaptation à la peinture.

3. Autre exemple, dans le The national curriculum in England (décembre 2014), il s'agit pour le Key stage 3 d'apprendre à lire des textes littéraires de façon critique " through knowing how language, including figurative language [...] presents meaning » (p. 83).

4. Selon les cas, l'auteur du commentaire établit de tels liens avec la « communauté académique des savoirs verticalement structurés » (Rayou \& Bautier, 2013, p. 32) ou demeure dans le champ des partages quotidiens.

5. C'est-à-dire les conflits d'interprétation, qui « prennent source dans ce qui fait confrontation conflictuelle pour les élèves entre les modes de socialisation scolaire et non scolaire » (Rayou \& Bautier, 2009, p. 103).

6. La moitié a travaillé sur un corpus du XX $\mathrm{X}^{\mathrm{e}}$ siècle, l'autre sur un corpus du XVII ${ }^{\mathrm{e}}$ siècle. Nous avons choisi des textes et des tableaux qui nous semblaient présenter un niveau de difficulté équivalent.

7. Nous revenons à cette distinction entre compréhension (saisie du sens inhérent) et interprétation (conception d'un sens afférent), très présente dans les attendus des enseignants que nous avons interrogés ; même s'ils ne considèrent pas toujours ces deux opérations comme successives ni indépendantes l'une de l'autre, ils ne les conçoivent généralement pas dans leur articulation (Reuter $\&$ Tauveron, 2001).

8. Ces références théoriques ne sont pas explicitées par les enseignants, mais elles nous permettent de comprendre et d'étiqueter leurs attendus.

9. Nous ne pouvons donner ici le détail des caractéristiques langagières qui nous ont permis de classer les commentaires selon leur proximité par rapport aux registres attendus des enseignants.

10. « L'iconographie est cette branche de l'histoire de l'art qui se rapporte au sujet ou à la signification des œuvres d'art, par opposition à leur forme » (Panofsky, p. 13). 


\section{RÉFÉRENCES}

Arasse, D. (2008). Le détail : pour une histoire rapprochée de la peinture. Paris, France : Flammarion.

Bautier, É., \& Rayou, P. (1 ${ }^{\text {re }}$ éd. 2009, $2^{\text {nde }}$ éd. 2013). Les inégalités d'apprentissage : programmes, pratiques et malentendus scolaires. Paris, France: Presses universitaires de France.

Bautier, É. et Rayou, P. (2013). La littératie scolaire : exigences et malentendus. Les registres de travail des élèves. Éducation et didactique, 7(2), 29-46. Rennes, France : Presses universitaires de Rennes.

Bernstein, B. (2007). Pédagogie, contrôle symbolique et identité. Théorie, recherche, critique. Sainte-Foy, Québec : Presses de l'Université Laval.

Daunay, B. (2004b). Le commentaire : exercice, genre, activité ? Cahiers Théodile (5), 49-61. Villeneuve d'Ascq, France : Université Charles de Gaulle - Lille 3.

Eco, U. (1976). La production des signes. Paris, France : Librairie générale française.

Eco, U. (1992). Les Limites de l'interprétation. Paris, France : Grasset.

Genette, G. (1982). Palimpsestes : la littérature au second degré. Paris, France : Éditions du Seuil.

Goodman, N. (1990). Langages de l'art: une approche de la théorie des symboles. Nîmes, France : J. Chambon.

Groupe $\mu$ (1992). Traité du signe visuel. Paris, France : Éditions du Seuil.

Jauss, H. R. (2005). Pour une esthétique de la réception. Paris, France : Gallimard.
Lahire, B. (1993). La raison des plus faibles : rapport au travail, écritures domestiques et lectures en milieux populaires. Lille, France : Presses universitaires de Lille.

Lahire, B. (2008). La raison scolaire : école et pratiques d'écriture entre savoir et pouvoir. Rennes, France : Presses universitaires de Rennes.

Langlade, G., \& Rouxel, A. (2004). Le sujet lecteur : lecture subjective et enseignement de la littérature. Rennes, France: Presses universitaires de Rennes.

Marin, L. (1971). Études sémiologiques. Écritures, peintures. Paris, France : Klincksieck.

Panofsky, E., \& Teyssèdre, B. T. (1967). Essais d'iconologie : thèmes humanistes dans l'art de la Renaissance (traduit par C. Herbette). Paris, France : Gallimard.

Reuter, Y. (2001). Comprendre, interpréter... en situation scolaire. Retour sur quelques problèmes. Dans C. Tauveron (dir.), Comprendre et interpréter le littéraire à l'école et au-delà (p. 69-79). Paris, France : INRP.

Reuter, Y. (1992). Comprendre, interpréter, expliquer des textes en situation scolaire. À propos d'Angèle. Pratiques (76), 7-25. Metz, France : CRESEF.

Rouxel, A. (1996). Enseigner la lecture littéraire. Rennes, France: Presses universitaires de Rennes.

Tauveron, C. (2001). Relations conjugales dans le couple infernal comprendre / interpréter. Dans C. Tauveron (dir.), Comprendre et interpréter le littéraire à l'école et au-delà (p. 5-24). Paris, France : INRP.

Waysbord-Loing, H. (2000). Limage dans l'enseignement des lettres, Rapport de l'inspection générale. Repéré à http://media.education.gouv.fr/file/99/6/5996.pdf 